Supporting information for

\title{
Tuning of Catalytic CO oxidation by Changing Composition of Rh- Pt Bimetallic Nanoparticles
}

\author{
Jeong Y. Park ${ }^{\dagger, \S}$, Yawen Zhang ${ }^{\dagger, \ddagger, \S}$, Michael Grass ${ }^{\dagger}$, Tianfu Zhang ${ }^{\dagger}$, and Gabor A. \\ Somorjai $^{\dagger} *$ \\ ${ }^{\dagger}$ Department of Chemistry, University of California, Berkeley, California 94720, and the \\ Chemical and Materials Sciences Divisions, Lawrence Berkeley National Laboratory, 1 \\ Cyclotron Road, Berkeley, California 94720 \\ ${ }^{\ddagger}$ College of Chemistry and Molecular Engineering, and the State Key Lab of Rare Earth \\ Materials Chemistry and Applications \& PKU-HKU Joint Lab in Rare Earth Materials and \\ Bioinorganic Chemistry, Peking University, Beijing 100871, China \\ ${ }^{\S}$ These authors contributed equally to this work.
}

* To whom correspondence should be addressed. E-mail: somorjai@berkeley.edu. 


\section{A. Size and Surface Structure of Rh/Pt bimetallic nanoparticles}

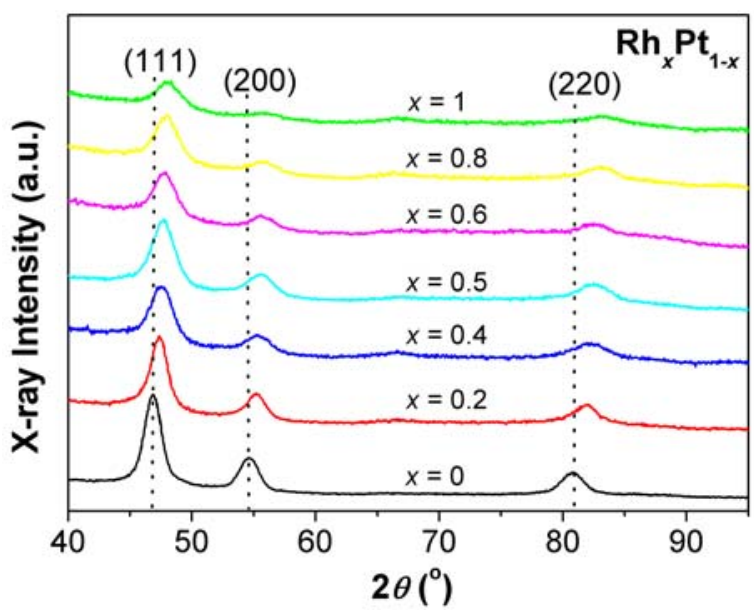

Figure 1S. XRD patterns of the $\mathrm{Rh}_{\chi} \mathrm{Pt}_{1-\chi}$ nanocrystals.

Powder X-ray diffraction (XRD) patterns were recorded on a Bruker D8 GADDS diffractometer using Co- $\mathrm{K}_{\alpha}$ radiation $(\lambda=1.79 \AA)$. Figure 1S shows the powder XRD patterns of various $\mathrm{Rh}_{x} \mathrm{Pt}_{1-x}$ nanocrystals. XRD results indicate that the (111) facets of the nanocrystals appear to be more preferential than (200) facets as the Rh composition increases. Figure 2S show size distribution histograms of the $\mathrm{Rh}_{\mathrm{x}} \mathrm{Pt}_{1-\mathrm{x}}$ nanocrystals: (a) $x=0$; (b) $x=0.2$; (c) $x=0.4$; (d) $x=0.5$; (e) $x=0.6$; (f) $x=0.8$. The TEM image and size distribution of Rh nanoparticles have been reported elsewhere ${ }^{1}$. Nanoparticle size and standard deviation is calculated by measuring 150 particles from TEM images. 


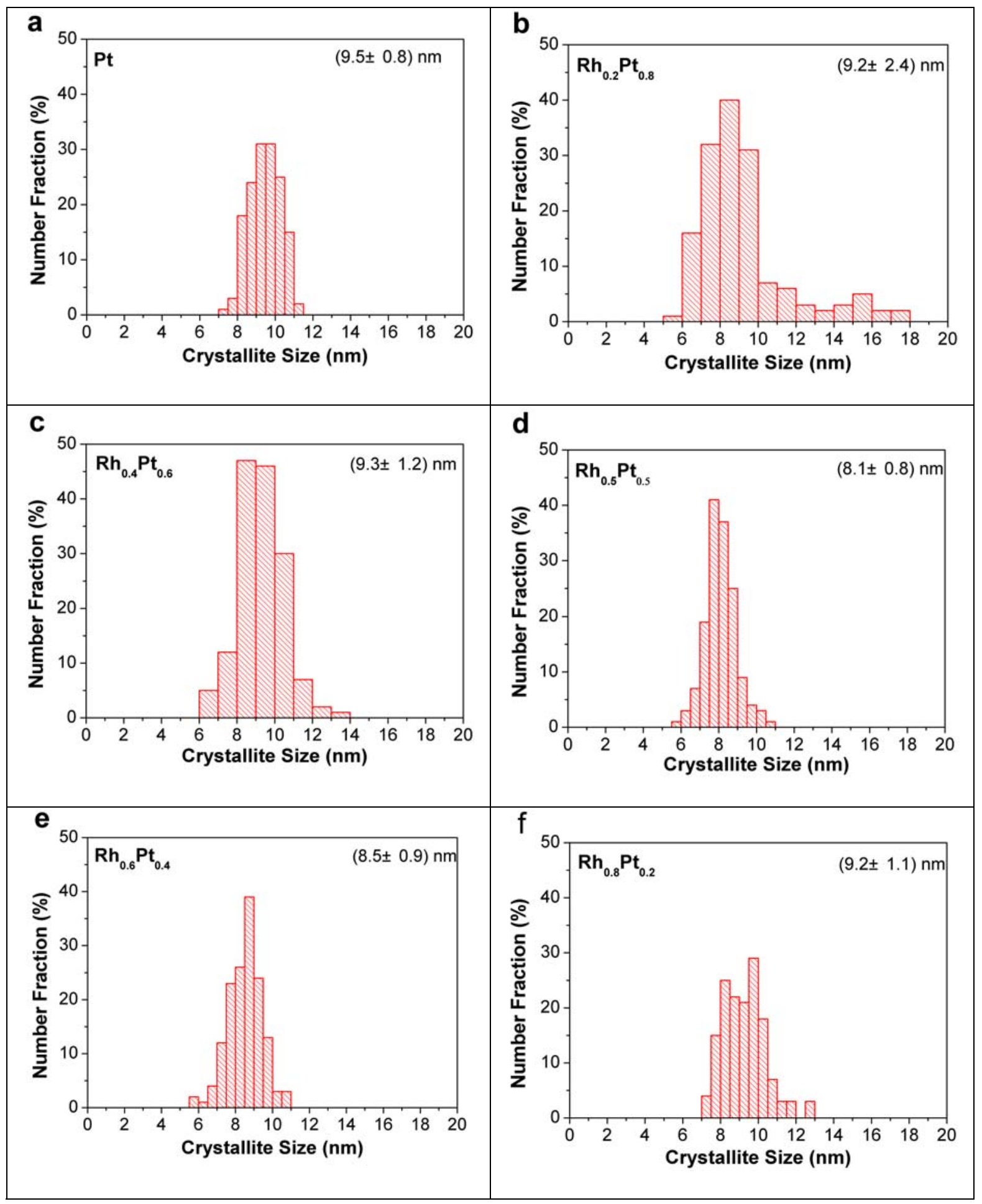

Figure 2S. Size distribution histograms of the $\mathrm{Rh}_{x} \mathrm{Pt}_{1-x}$ nanocrystals: (a) $x=0$; (b) $x=0.2$; (c) $x=$ 0.4 ; (d) $x=0.5$; (e) $x=0.6$; (f) $x=0.8$. 


\section{B. Influence of CO oxidation on chemical composition and morphology of nanoparticles -}

XPS and SEM studies after the reactions.
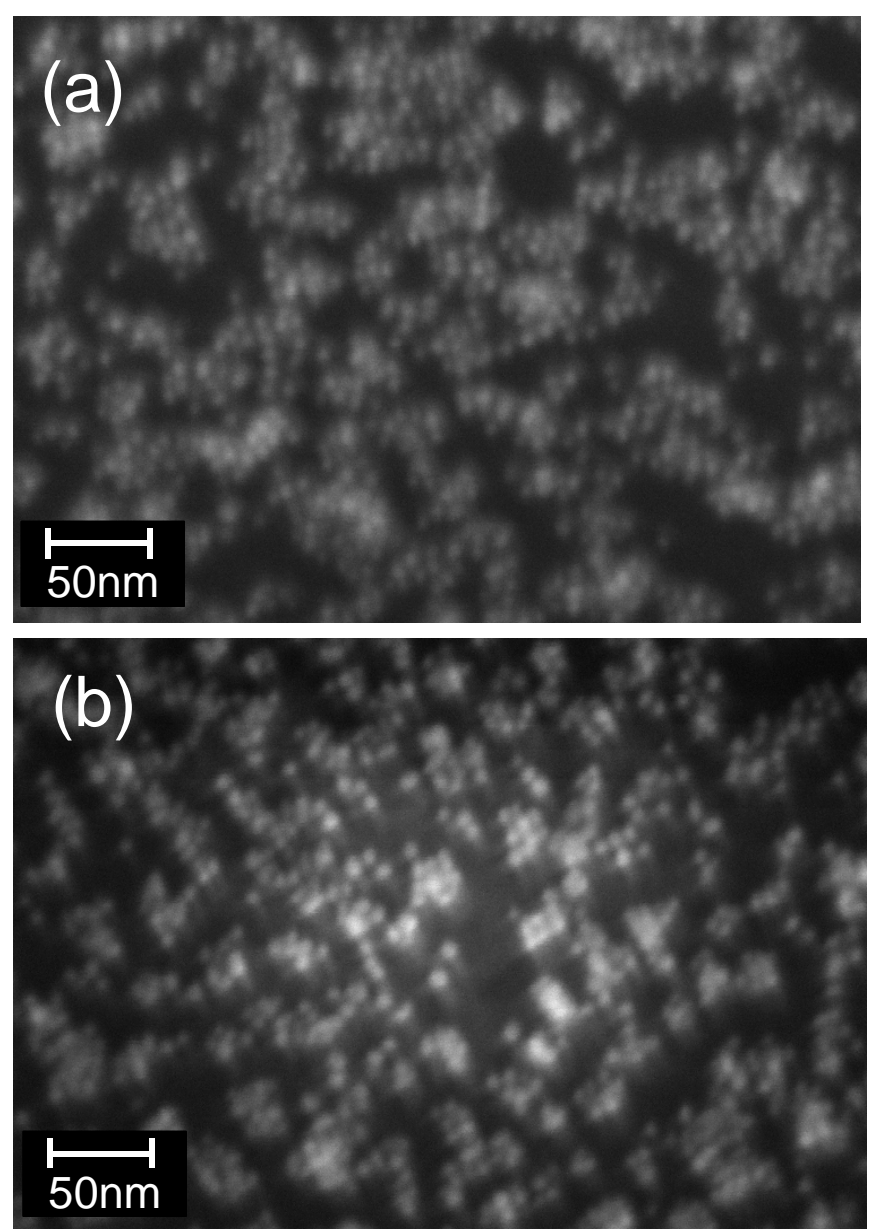

Figure 3S. SEM image of $\mathrm{Pt}_{0.6} \mathrm{Rh}_{0.4}$ on silicon (a) before and (b) after CO oxidation up to $220^{\circ} \mathrm{C}$.
The change of
morphology and
composition of bimetallic
nanoparticles after CO
oxidation up to $220{ }^{\circ} \mathrm{C}$
was checked with XPS
and SEM. Figure $3 \mathrm{~S}$

shows the SEM image of $\mathrm{Rh}_{0.4} \mathrm{Pt}_{0.6}$ nanocrystals on a silicon surface (a) before and (b) after CO oxidation. The resolution of $\operatorname{SEM}(\sim \quad 1 \quad \mathrm{~nm})$ permitted us to reveal individual nanoparticles. The size of the nanoparticles based on the SEM image was $9 \pm 1 \mathrm{~nm}$, close to the values (9.3 $\mathrm{nm}$ ) determined with the

TEM measurement. After the reaction, we did not observe any significant change in the SEM images, indicating the absence of agglomeration of nanoparticles. 


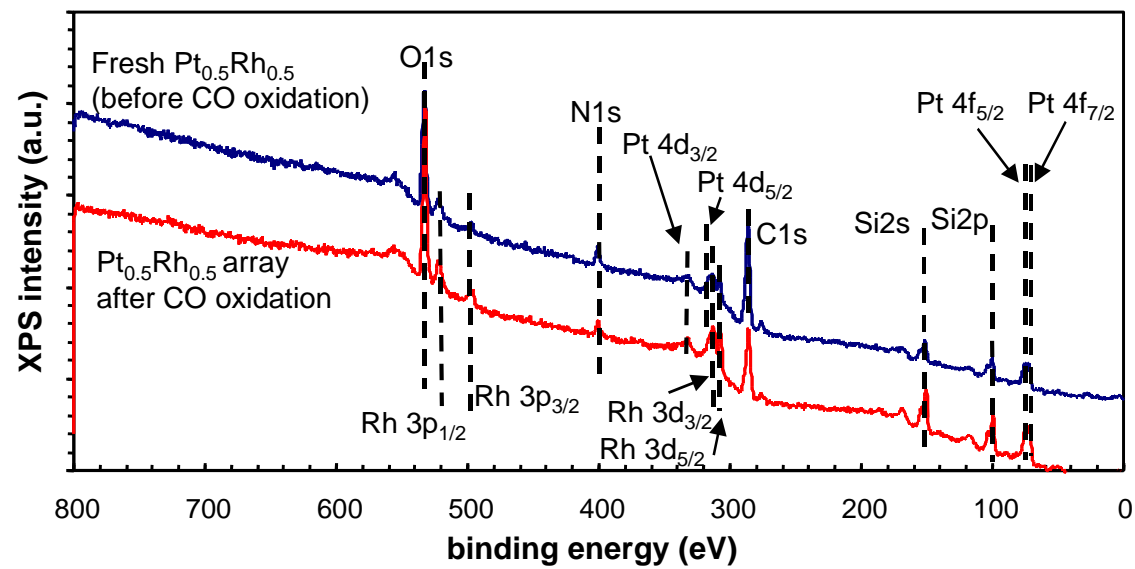

Figure 4S. XPS of $\mathrm{Pt}_{0.5} \mathrm{Rh}_{0.5}$ on silicon (a) before and (b) after CO oxidation up to $220{ }^{\circ} \mathrm{C}$.

Figure 4S shows the XPS results of $\mathrm{Rh}_{0.5} \mathrm{Pt}_{0.5}$ before and after $\mathrm{CO}$ oxidation. Here, $\mathrm{Rh}$ composition of 0.5 refers to the molar ratio of $\mathrm{Rh}(\mathrm{acac})_{3}$ to total amount of $\mathrm{Rh}(\mathrm{acac})_{3}$ and $\mathrm{Pt}(\mathrm{acac})_{2}$. The composition of the fresh sample was determined to be $\mathrm{Rh}_{0.49} \mathrm{Pt}_{0.51}$ based on composition analysis with XPS, as shown in Fig. 4S. After reaction, XPS measurements indicated a composition of $\mathrm{Rh}_{0.46} \mathrm{Pt}_{0.54}$. In other bimetallic nanoparticles, we found that the relative composition between Pt and Rh was not changed within 3\% after CO oxidation. We also found that the intensity of N1s and C1s peaks decreased, suggesting that some PVP was removed. The ratio of compensated area (compensated area is the area divided by the XPS sensitivity factor of the peak.) of the C1s peak to the sum of the compensated area of Rh3d and Pt4f decreased from 12.2 to 6.7 after CO oxidation. Likewise, the ratio of compensated area of the N1s peak to that of sum of Rh3d and Pt4f decreased from 3.4 to 1.2 after the reaction. This result suggests that carbonaceous and nitrogenous species in the capping layers are partially decomposed during CO oxidation.

\section{References}

1. Zhang, Y. et al. One-step Polyol Synthesis and Langmuir-Blodgett Monolayer Formation of Size-tunable Monodisperse Rhodium Nanocrystals with Catalytically Active (111) Surface Structures. Journal of Physical Chemistry C 111, 12243-12253 (2007). 